\title{
SIMPLIFIED ANALYSIS PROCEDURE ON DAMPED VIBRATION EQUIVALENT TO LOVE WAVES IN DOUBLE-LAYERED VISCO-ELASTIC MEDIA
}

\author{
By Tatsuo OHMACHI*, Shigeaki MORICHI** and Takumi TOSHINAWA***
}

\begin{abstract}
Love weve propagation is essentially equivalent to shear vibration of layered media. On this basis, with the introduction of a common assumption which has been employed in vibration analysis of structures, a simplified procedure is formulated to evaluate ground motion resulting from Love wave propagation in visco-elastic media. As the procedure allows us to use vibration mode shapes of an elastic system without viscous damping, we can easily evaluate response of a visco-elastic layered system in almost the same way as for a damped single degree of freedom system. In addition, a new index called a modal response factor is presented to demonstrate its usefulness and some comments are discussed for laboratory experiments.

Keywords: Love wave, shear vibration, visco-elasticity, layered media
\end{abstract}

\section{INTRODUCTION}

There are many reasons for a civil engineer to think it very important to predict earthquake ground motion with a satisfactory level of accuracy. First, for example, earthquake ground motion is regarded as an input excitation to which any civil engineering structures such as dams and bridges are forced to respond. Since earthquake response of a structure varies appreciably with a change in characterisics of the input excitation, earthquake ground motion plays a prominent role in earthquake resistant design of the structure. Next, various functions of modern cities have been supported by the lifeline network systems, and most lifeline facilities mainly consist of underground structures to which earthquake-induced ground strain has caused a lot of serious damage. Taking into account that almost all large cities in Japan are located on sedimentary layers, accurate prediction or characterization of earthquake ground motion especially associated with the surface wave components must be and has been one of the most fundamental subjects of seismic disaster mitigation.

Some parts of analytical and experimental difficulties in treatment of the surface waves can be attributed to boundary conditions imposed on a layered structure, because horizontal length of layered media have usually been supposed to be infinite. But if we can efficiently realize surface waves by means of layered media of finite length, the treatment will be simplified to a certain extent. From this point of view, the authors demonstrated in their previous paper ${ }^{1)}$ that shear vibration of an elastic surface layer of finite length is fully characterized by Love wave propagation. In one sense, it is a self-evident matter because

* Member of JSCE, Dr. Eng., Associate Professor, Tokyo Institute of Technology (4259, Nagatsuta, Midori-ku, Yokohama)

** Member of JSCE, Dr. Eng. , Associate Professor, Science University of Tokyo (2641, Yamazaki Higashi Kameyama, Noda-shi, Chiba)

*** Member of JSCE, Research Associate, Tokyo Institute of Technology (4259, Nagatsuta, Midori-ku, Yokohama) 
vibration and wave propagation are equivalent in principle. But, if use is made of the equivalence between wave propagation and vibration motion, ground motion caused by propagation of surface waves may be evaluated much easier than before by using vibration analysis technique to which civil engineers have been much accustomed.

For these reasons, in this paper, the authors intend to derive some of fundamental shear vibration characteristics of visco-elastic layered media which do not fail to serve greatly in evaluation or characterization of Love wave propagation in the same media.

\section{REDUCTION OF VISCO-ELASTIC MODE SHAPES TO ELASTIC ONES}

Let us consider two-dimensional double-layered systems of viscoelastic media and take Cartesian coordinates for them, as shown in Fig. 1. In Fig. 1 (b), $L$ is length of the layers bounded by vertical side walls and $H$ is uniform depth of the upper layer. As for visco-elasticity of the media, it is assumed to be of the Voigt-type. The essential properties involed in plane shear distortion in the direction orthogonal to the $x-z$ plane ( $y$ direction) are shear modulus $G$, viscosity coefficient $\eta$ and mass density $\rho$ of each layered medium. The free vibration equation of motion of the system shown in Fig. $1(\mathrm{~b})$ is expressed by

$$
\rho \frac{\partial^{2} u}{\partial t^{2}}=G\left(\frac{\partial^{2} u}{\partial x^{2}}+\frac{\partial^{2} u}{\partial z^{2}}\right)+\eta\left(\frac{\partial^{3} u}{\partial t \partial x^{2}}+\frac{\partial^{3} u}{\partial t \partial z^{2}}\right)
$$

where $u$ denotes horizontal displacement in the $y$ direction. In this paper, subscripts 1 and 2 are assinged to refer to the upper and lower layers respectively, and shear wave velocities of each layer are assumed to be $v_{1}<v_{2}$ for simplicity. Boundary conditions imposed on $u_{1}$ and $u_{2}$ are;

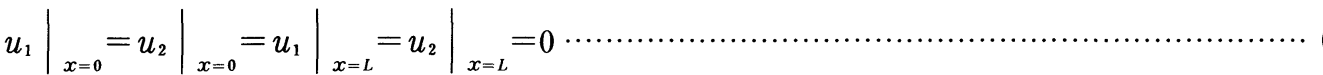

$$
\begin{aligned}
& \left.u_{1}\right|_{z=0}=\left.u_{2}\right|_{z=0}, \text { and }\left.u_{2}\right|_{z=-\infty}=0 \\
& \left.\frac{\partial u_{1}}{\partial z}\right|_{z=H}=0, \quad \text { and }\left.\quad\left(G_{1} \frac{\partial u_{1}}{\partial z}+\eta_{1} \frac{\partial^{2} u_{1}}{\partial t \partial z}\right)\right|_{z=0}=\left.\left(G_{2} \frac{\partial u_{2}}{\partial z}+\eta_{2} \frac{\partial^{2} u_{2}}{\partial t \partial z}\right)\right|_{z=0} \cdots
\end{aligned}
$$

In comparison with a case of an elastic layered system, it is usually much more tedious to solve Eq. ( 1 ) under the boundary conditions in Eps. (2) to (4), and vibration mode shapes associated with the solution are of a complex form made up of both real and imaginary components. Thus, for practical application, it will be very useful to obtain a simplified solution having a satisfactory level of accuracy.

For this purpose, it is helpful to recall the damping orthogonality ${ }^{2)}$ which permits us to use undamped vibration mode shapes instead of damped ones in dynamic response analysis of a lumped parameter system. One of the simplest examples which satisfy the damping orthogonality is so-called Rayleigh damping whose damping matrix is expressed by a linear combination of mass and stiffness matrices. In application of the mode superposition procedure, it is a common practice to evaluate structural earthquake response by means of the undamped mode shape vectors and damped frequencies, assuming the damping orthogonality. A similar simplification of vibration modes will be applied to the visco-elastic layered system concerned. Vibration modes of the corresponding elastic system can readily be obtained by setting each viscosity coefficient equal to zero, giving

$$
u_{1}(x, z, t)=a_{0} \sin k x \cos \zeta_{1}(z-H) e^{i \omega t}
$$


$u_{2}(x, z, t)=a_{0} \cos \zeta_{1} H \sin k x e^{\zeta_{2} z} e^{i \omega t}$

where $a_{0}$ is an arbitrary constant, and

$$
\begin{aligned}
& \zeta_{1}=\sqrt{k_{1}^{2}-k^{2}}, \zeta_{2}=\sqrt{k^{2}-k_{2}^{2}}, \quad \text { and } \\
& k_{2}\left(=\frac{\omega}{v_{2}}\right)<k\left(=\frac{n \pi}{L}\right)<k_{1}\left(=\frac{\omega}{v_{1}}\right), \quad n=1,2,3
\end{aligned}
$$

Similarly to Rayleigh damping, let the viscosity be expressed by a linear combination of the mass density and shear modulus,

$$
\eta_{1}=a \rho_{1}+b G_{1} \quad \text { and } \quad \eta_{2}=a \rho_{2}+b G_{2}
$$

where $a$ and $b$ are constants. Then, introducing the vibration modes expressed by Eqs. (5) and (6) into Eq. (1), and imposing the boundary conditions in Eqs. (2) to (4) lead to conditions under which the vibration mode shapes of the visco-elastic system become identical to those of the elastic system, with a result

$$
a=0 \text { or } v_{1}=v_{2}
$$

The second condition in Eq. ( 9 ) is abandoned because of the previously mentioned assumption $v_{1}<v_{2}$. The first condition is equivalent to assume that both of the layered media have a commonly specified value of the attenuation factor $Q^{3)}$ being inversely proportional to frequency, as can be seen later in Eq. (10) . Moreover, it should be added that if the condition holds, in other words if the viscosity coefficient is proportional to the shear modulus, complex circular frequency $\omega^{*}$ and modal damping ratio $h$ of the system in this case are simply given by

$$
i \omega^{*}=-h \omega+i \omega \sqrt{1-h^{2}} \equiv-h \omega+i \omega_{d} \quad \text { and } \quad h=\frac{\eta_{1} \omega}{2 G_{1}}=\frac{\eta_{2} \omega}{2 G_{2}}
$$

in which $\omega$ denotes the circular frequency of the elastic system given as a solution of the following characteristic equation.

$$
\tan \sqrt{k_{1}^{2}-k^{2}} H=\frac{G_{2}}{G_{1}} \sqrt{\frac{k^{2}-k_{2}^{2}}{k_{1}^{2}-k^{2}}}
$$

In some cases, Eq. (11) gives plural sets of $k_{1}$ and $k_{2}$ for a single value of $k(=n \pi / L)$. In such a case, among plural frequencies given by $\omega=k_{1} v_{1}=k_{2} v_{2}$, a mode number m equal to 0 is usually assigned to the lowest frequency, m equal to 1 is assigned to the next higher frequency, etc. As a vibration shape along the $z$ axis is determined by a set of $k_{1}, k_{2}$ and $k$ as seen in Eqs. ( 5 ) through ( 7 ), the shape in the mode m will be denoted by $W_{m}(z)$ in the following section.

From the frequency expression in Eq. (10), it is obvious that the frequency of realistic soil layers whose attenuation factor $Q(=$ $1 / 2 h$ ) is usually greater than 5 , is almost equal to that of an elastic system having the same mass density and shear modulus. It is very important to note, in passing, that the foregoing formulation on shear vibration characteristics of horizontal layers also applies to Love waves in visco-elastic layers shown in Fig. 1 (a) with a slight modification. The modification is attributed to the finite length of the layers,

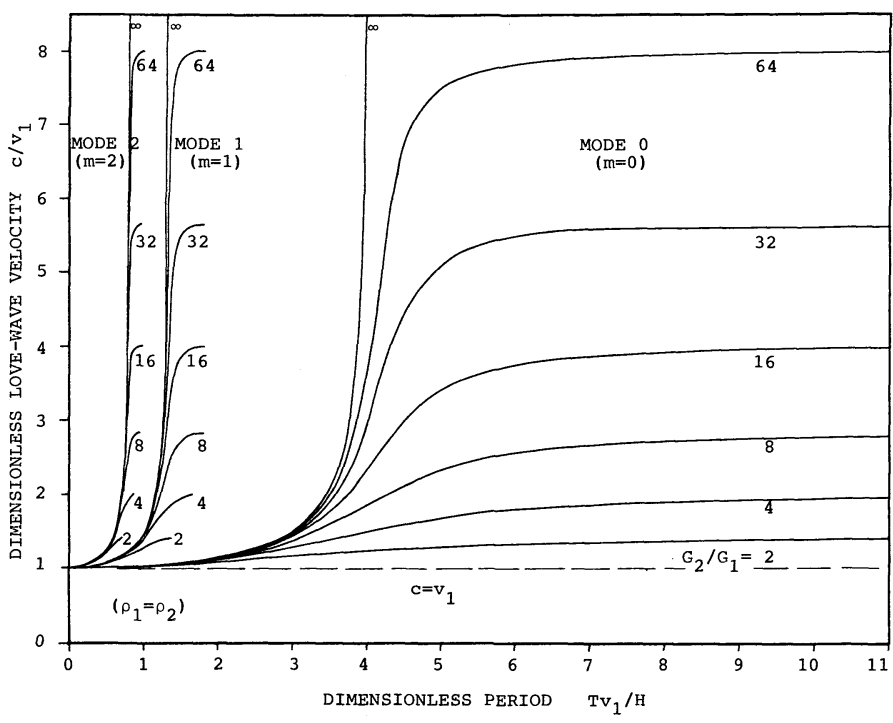

Fig. 2 Phase Velocity plotted against Period. 
while infinite length assumption has usually been adopted in formulation of Love wave characteristics.

In Eq. ( 7 ), the number $k$ can be regarded as a wave number counted along the $x$ axis. When the layer length $L$ is fixed, the wave number $k$ and the circular frequency $\omega$ can take only discrete values due to the integer $n$. But when the length $L$ is continuously varied in the interval $0<L<\infty$ with $n$ set equal to $2, k$ and $\omega$ will also vary continuously. Consequently, if we define a phase velocity $c$ and a period $T$ as

$c=\omega / k$ and $T=2 \pi / \omega$

and plot their relation, we will obtain a continuous curve. In Fig. 2 are shown such curves for the lowest three modes of shear vibration of the layered system with various shear modulus ratios $G_{2} / G_{1}$. It should be noted here that Fig. 2 which originally shows shear vibration characteristics of the layered system, shows the dispersion characteristics of Love waves as well. This is due to essential equivalence between these two characteristics. In fact, not only the equation of motion in Eq. (1) but also the boundary conditions in Eqs. (2) to (4) are satisfied by Love wave components whose wave length is an integer multiple of the length $L$. This equivalence could serve us to evaluate earthquake ground motion associated with Love wave propagation by means of a procedure usually adopted for shear vibration analysis of layered media of finite length.

\section{RESPONSE TO AN IMPULSIVE LOADING}

The equation of motion for a horizontally layered system subjected to a dynamic loading $f(x, z, t)$ is generally expressed by

$$
\rho(z) \frac{\partial^{2} u}{\partial t^{2}}=G(z)\left(\frac{\partial^{2} u}{\partial x^{2}}+\frac{\partial^{2} u}{\partial z^{2}}\right)+\eta(z)\left(\frac{\partial^{3} u}{\partial t \partial x^{2}}+\frac{\partial^{3} u}{\partial t \partial z^{2}}\right)+f(x, z, t)
$$

where mass density, viscosity coefficient and shear modulus are assumed to vary with depth, and denoted by $\rho(z), \eta(z)$ and $G(z)$, respectively. It is apparent from derivation in the previous section that if the viscosity coefficient is proportional to the shear modulus,

$$
\eta(z)=b G(z)
$$

in which $b$ is a constant, a particular solution of Eq. (13) is given by

$$
\begin{aligned}
& u(x, z, t)=\sum_{m} \sum_{n} U_{n}(x) W_{m}(z) q_{m n}(t) \\
& U_{n}(x)=\sin k x \quad(0 \leqq x \leqq L) \\
& W_{m}(z)= \begin{cases}\cos \zeta_{1}(z-H) & (0 \leqq z \leqq L) \\
\cos \zeta_{1} H e^{\zeta_{2} z} & (z<0)\end{cases}
\end{aligned}
$$

Substituting Eqs. (14) and (15) into Eq. (13), multiplying on both sides of the resulting equation by $G(z) U_{n},(x) W_{m},(z) / \rho(z)$ and integrating it in the intervals $0<x<L$ and $-\infty<z<H$, lead to an uncoupled equation of motion formally equivalent to that of a single degree of freedom system, with a result

$$
\ddot{q}_{m n}(t)+2 h \omega \dot{q}_{m n}(t)+\omega^{2} q_{m n}(t)=F_{m n}(t) / M_{m n}
$$

where

$$
\begin{aligned}
& F_{m n}(t)=\int_{-\infty}^{H} \int_{0}^{L} \frac{G(z)}{\rho(z)} U_{n}(x) W_{m}(z) f(x, z, t) d x d z \\
& M_{m n}=\int_{-\infty}^{H} \int_{0}^{L} G(z) U_{n}^{2}(x) W_{m}^{2}(z) d x d z \ldots \ldots \ldots \ldots \ldots \ldots \ldots \ldots \ldots
\end{aligned}
$$

In the above process to derive Eq. (18), advantage has been taken of the orthogonality of the mode shape functions ${ }^{4)}$ expressed by

$$
\begin{aligned}
& \int_{0}^{L} U_{n}(x) U_{n} \cdot(x) d x=0 \quad\left(n \neq n^{\prime}\right) \\
& \int_{-\infty}^{H} G(z) W_{m}(z) W_{m} \cdot(z) d z=0 \quad\left(m \neq m^{\prime}\right)
\end{aligned}
$$

When a unit impulse is applied at a point $\left(x_{0}, z_{0}\right)$ pertaining to the upper layer at time $t=0$, the loading function is expressed by using the Dirac delta function $\delta(\cdot)$ as 
$f(x, z, t)=\delta\left(x-x_{0}\right) \delta\left(z-z_{0}\right) \delta(t)$

Substituting Eq. (22) into Eq. (19), putting for simplicity $\rho_{1}=\rho_{2}=\rho$, and performing integrations in Eqs.

(19) and (20) lead to

$$
\begin{aligned}
& F_{m n}(t)=\frac{G_{1}}{\rho} \sin k x_{0} \cos \zeta_{1}\left(z_{0}-H\right) \delta(t) \ldots \ldots \ldots \ldots \ldots \ldots \ldots \ldots \ldots \ldots \ldots \ldots \ldots \ldots \ldots \\
& M_{m n}=\frac{G_{1} H L}{4 \zeta_{1} H \tan \zeta_{1} H}\left\{\left(\frac{G_{2}}{G_{1}}\right)^{2} \cos ^{2} \zeta_{1} H+\zeta_{1} H \tan \zeta_{1} H+\sin ^{2} \zeta_{1} H\right\}
\end{aligned}
$$

Hence modal response of the layered system to the unit impulse can be readily obtained by analogy with the unit impulsive response of a single degree of freedom system, giving

$$
q_{m n}(t)=\frac{4}{\rho H L} \frac{G_{1} H A_{L}}{\omega_{d}} \sin k x_{0} \cos \zeta_{1}\left(z_{0}-H\right) e^{-h \omega t} \sin \omega_{d} t
$$

where

$$
A_{L}=\frac{\zeta_{1} H}{G_{1} H} \frac{\tan \zeta_{1} H}{\left(G_{2} / G_{1}\right)^{2} \cos ^{2} \zeta_{1} H+\zeta_{1} H \tan \zeta_{1} H+\sin ^{2} \zeta_{1} H}
$$

A unit impulsive response in the geometrical coordinates can then be obtained by the sum of the modal responses. When the response is observed at a point $(x, z)$ pertaining to the upper layer, it will be

$$
u(x, z, t)=\sum_{m} \sum_{n} \frac{4}{\rho H L} \frac{G_{1} H A_{L}}{\omega_{d}} \sin k x_{0} \cos \zeta_{1}\left(z_{0}-H\right) \sin k x \cos \zeta_{1}(z-H) e^{-h \omega t} \sin \omega_{d} t
$$

In Eq. (27), symmetry between each pair of coordinates $(x, z)$ and $\left(x_{0}, z_{0}\right)$ can be regarded as a statement of Maxwell's reciprocal theorem.

It should be noted here that $A_{L}$ defined by Eq. (26) is the same that referred to as medium response or amplitude response factor of Love waves ${ }^{5)}$.7). From Eqs. (24) and (26), it is obvious that $A_{L}$ derives from the integration for $M_{m n}$ which is independent of loading. Making $A_{L}$ dimensionless by multiplying $G_{1} H$, we will call it a medium response factor. In Fig. 3, the medium response factor is plotted against a dimensionless period $T v_{1} / H$ for the lowest three modes of vibration, from which we can easily draw some of findings analytically obtained in the early study on Love wave in multilayered media.

In order to evaluate contribution of modal response to total response, it is much more useful to discuss in detail the medium response factor after dividing it by the modal frequency, as may be seen from Eq. (25) or (27). Since the damped modal frequency $\omega_{d}$ is practically equal to the undamped modal frequency $\omega$, a dimensionless factor $G_{1} v_{1} A_{L} / \omega$ defined for elastic media can also serve to indicate modal contribution to the total response of visco-elastic media. Thus the factor $G_{1} v_{1} A_{L} / \omega$ will be called a modal response factor in this paper, and it is plotted against the dimensionless period as well as against the dimensionless wave length in Fig. 4 and 5, respectively.

It is apparent from Fig. 4 that when the shear modulus ratio $G_{2} / G_{1}$ is infinite, a peak period at which the modal response become its maximum is simply given by $T_{0}=4 H / v_{1}$. Note that the peak period becomes

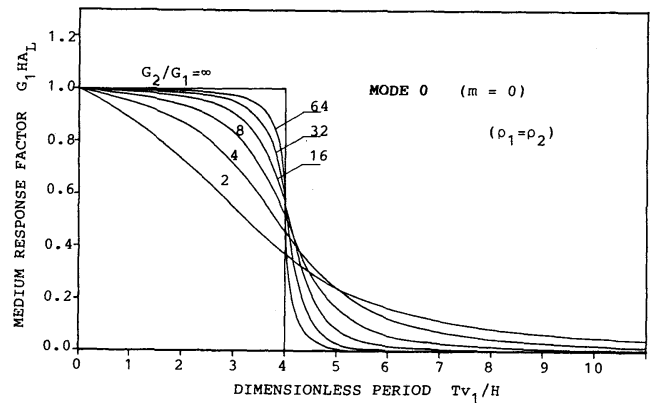

(a)

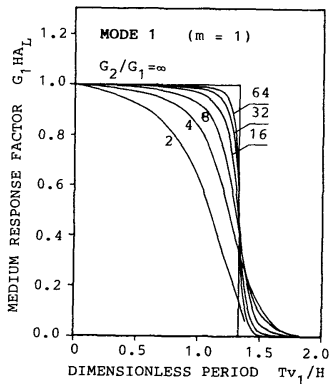

(b)

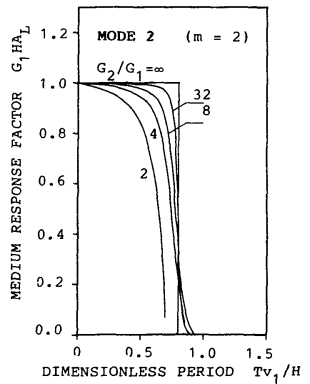

(c)

Fig. 3 Medium Response Factor plotted against Period 


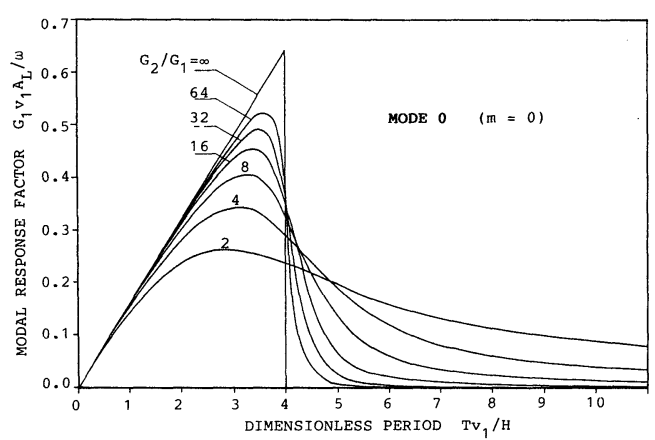

(a)

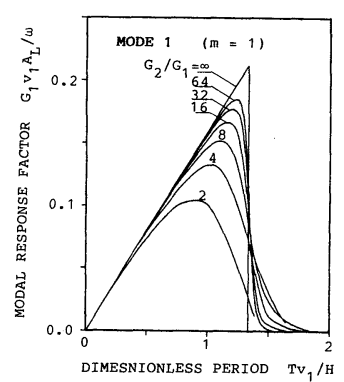

(b)

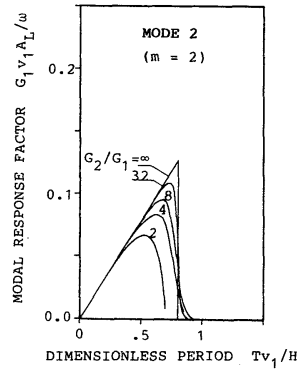

(c)

Fig. 4 Modal Response Factor plotted against Period.

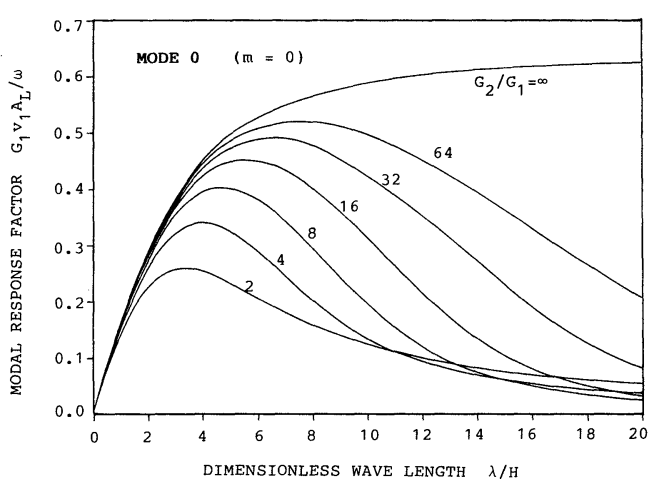

(a)

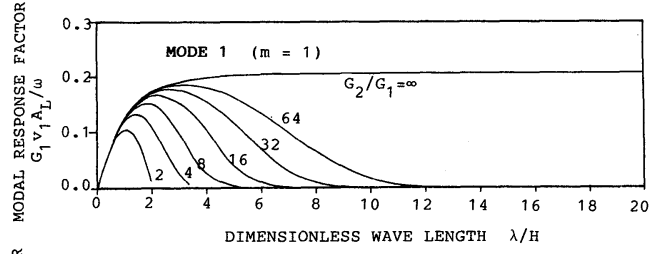

(b)

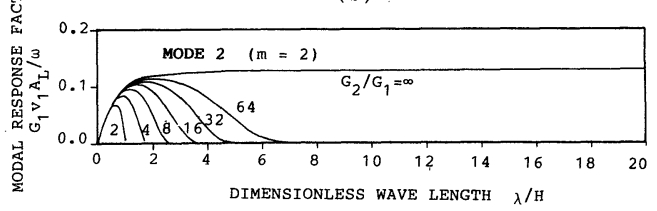

(c)

Fig. 5 Modal Response Factor plotted against Wave Length.

shorter than $T_{0}$ with a decrease in the shear modulus ratio. These are consistent with the so-called quarter wave-length law of Love waves ${ }^{8)}$. Moreover, when the shear modulus of the upper layer remains constant, response amplitude decreases with a decrease in the shear modulus ratio. As for response of higher modes, contribution to the total response decreases by roughly $1 / 3,1 / 5, \cdots$ of the fundamental mode with an increase in mode number $\mathrm{m}$. This is also consistent with the Love wave characteristics described elsewhere ${ }^{8)}$. The modal response characteristics illustrated in Fig. 4 and 5 will be useful in practical evaluation of ground motion caused by Love wave propagation, since the motion can be evaluated by summing up the modal responses appropriately as stated earlier.

\section{DISCUSSION FOR EXPERIMENTS}

Well-designed experiments serve so greatly for us to understand the before-mentioned equivalence between Love wave propagation and shear vibration of a surface layer, that some comments for the experiments are added in the following.

In case of the shear modulus ratio $G_{2} / G_{1}$ being infinite, the equations and resulting characteristics mentioned in the foregoing sections are drastically simplified and provide a useful guide to vibration experiments. In this case, the modal response in free vibration is reduced to

$$
q_{m n}(t)=\frac{4}{\rho H L \omega_{d}} \sin k x_{0} \sin s z_{0} e^{-h \omega t} \sin \omega_{d} t
$$

where $k=n \pi / L, \quad s=(2 m+1) \pi /(2 H)$, and

$$
\omega_{d}=\omega \sqrt{1-h^{2}}
$$




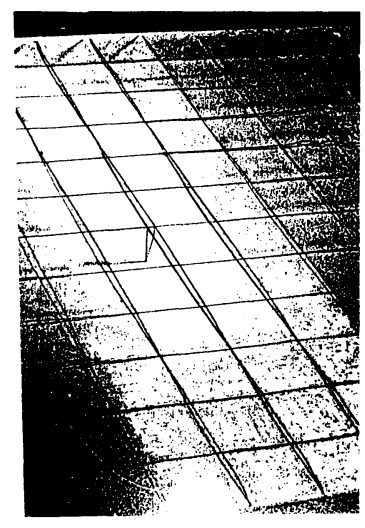

(a) at $13.0 \mathrm{~Hz}$

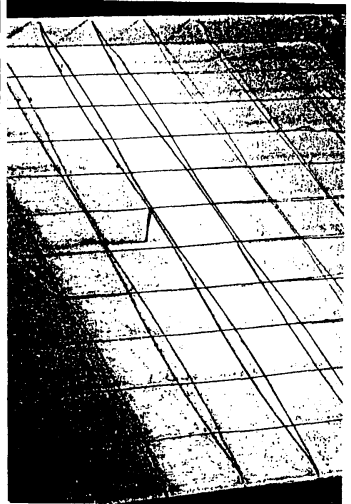

(b) at $13.8 \mathrm{~Hz}$

Photo 1 Standing Waveforms observed in Experiments.

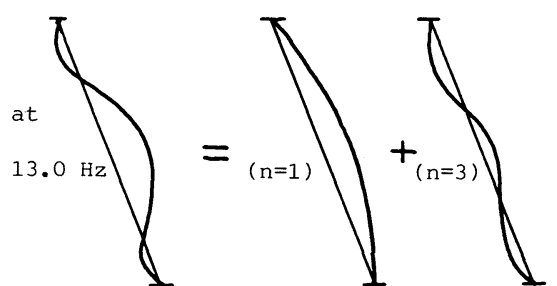

(a)

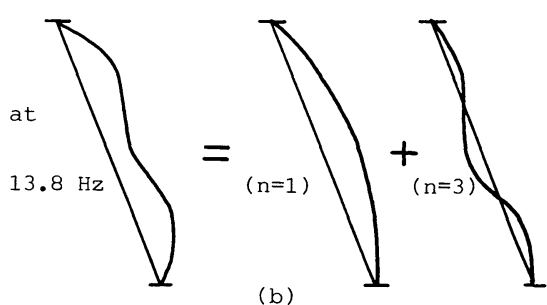

Fig. 6 Decomposition of Observed Waveforms.

$$
\omega=v_{1} \sqrt{k^{2}+s^{2}} \text { and } h=\frac{\eta_{1} \omega}{2 G_{1}}
$$

It should be noticed that the above expressions are valid for any homogeneous layer with visco-elasticity of the Voigt-type resting on a rigid basement, because the damping proportionality in Eq. (16) is always satisfied implicitly.

It has been well known that a frequency response function and an impulsive response function are one of the Fourier transform pairs. Thus, for a sinusoidal excitation applied at the point $\left(x_{0}, z_{0}\right)$, the modal amplitude $q_{m n}^{*}$ at frequency $\omega_{0}$ is directly obtained by calculating the Fourier transform of Eq. (28), with a result

$$
q_{m n}^{*}=\frac{4}{\rho H L} \frac{1}{\sqrt{\left(\omega-\omega_{0}\right)^{2}+\left(2 h \omega_{0}\right)^{2}}}
$$

In practice, however, it is not easy to apply a concentrated loading at a point inside the layer, whether the loading is impulsive or sinusoidal. Instead, it is much easier to give rise to vibration modes by means of a shaking table. When the layer mounted on a shaking table is put into vibration by sinusoidal table motion $u_{0} \exp \left(i \omega_{0} t\right)$, the loading function is given in the form

$$
f(x, z, t)=\rho u_{0} \omega_{0}^{2} e^{i \omega_{0} t}
$$

In this case, the definite integrations for $F_{m n}(t)$ and $M_{m n}$ result in

$$
F_{m n}(t)=\frac{2\left\{1-(-1)^{n}\right\}}{(2 m+1) n \pi^{2}} \rho H L u_{0} \omega_{0}^{2} e^{i \omega_{0} t} \text { and } M_{m n}=\frac{\rho H L}{4}
$$

By analogy with sinusoidal vibration of a single degree of freedom system, the modal amplitude $q_{m n}^{*}$ is found to be

$$
q_{m n}^{*}=\frac{8\left\{1-(-1)^{n}\right\}}{(2 m+1) n \pi^{2}} \frac{u_{0} \omega_{0}^{2}}{\sqrt{\left(\omega-\omega_{0}\right)^{2}+\left(2 h \omega_{0}\right)^{2}}}
$$

Note that two expressions for $q_{m n}^{*}$ in Eqs. (31) and (34) have a formal resemblance, but that there is a significant difference between them; that is, the factor $\left\{1-(-1)^{n}\right\} /(2 m+1) n$ is present in Eq. (34). This implies that, in vibration experiments using a shaking table, vibration modes corresponding to even numbers of $\mathrm{n}$ are hardly induced, and that higher modes are more difficult to be observed than in experiments applying a concentrated loading. Thus, one who intends to observe higher modes of vibration should be very careful in the experiments, otherwise he is likely to misunderstand them.

Photo 1 shows two examples of standing waveforms observed in a series of viration experiments using a 
shaking table. A description on this experiments such as material properties and dimension of the specimen can be seen in our previous paper ${ }^{1)}$. At a first look, the waveform in Photo 1(a) appears to show a vibration shape in the mode for $m=0$ and $n=1$, but it should be noticed that the middle part is appreciably longer in wave length than side parts. The waveform shown in ( $b$ ) looks a little strange because there is no node showing zero displacement between the two clearly observable antinodes. Each of these waveforms can roughly be decomposed into two vibration mode shapes, as shown in Fig. 6 . Note in Fig. 6 that both observed waveforms are made up of the same two components, but that the vibration mode shape for $n=3$ is reversed one another. Hence it is reasonable to think that a modal frequency for $m=0$ and $n=3$ should lie between the two frequencies $13.0 \mathrm{~Hz}$ and $13.8 \mathrm{~Hz}$. But a purified vibration shape in this mode can hardly be observed even just at the modal frequency because there will be a considerable amount of contribution of the fundamental mode $(n=1)$ to the total response, unless damping of the medium is very low and the modal frequencies are wide apart each other.

\section{CONCLUSION}

To simplify a tedious process of ground motion evaluation associated with Love wave propagation or shear vibration during earthquakes, free vibration and impulsive response of double-layered visco-elastic media have been investigated, with the following conclusions:

(1) If it is permitted to assume that every layered medium has a commonly specified value of the attenuation factor $Q$ which is inversely proportional to the frequency, the evaluation process is drastically simplified because vibration mode shapes of a visco-elastic layered structure become identical to those of the corresponding elastic structure.

(2) Even if viscous damping is involved in elastic media, the frequency of the layered structure is practically equal to that of the purely elastic structure. Thus, all we have to do in the evaluation is to take account of amplitude decay with time and space, which can be done in almost the same way as a response analysis for a single degree of freedom system.

(3) Instead of the medium response, a newly defined factor which is tentatively referred to as a modal response factor has been presented here. The factor has proved to be useful in direct evaluation of modal contribution to total response or spectral amplitude of impulsive response.

(4) Careless observation of vibration mode shapes is likely to lead us to misunderstanding of higher modes. This is mainly due to the fact that the modal contribution usually tends to decrease rapidly for the higher mode. This should be beared in mind especially in a vibration experiment using a shaking table.

(5) After all, since there is essential equivalence between Love wave propagation and shear vibration of layered media, a lot of Love wave characteristics can readily be derived from a viewpoint of vibration analysis of structures.

\section{REFERENCES}

1) Morichi, S., Ohmachi, T. and Toshinawa, T. : Vibration Modes Characterized by Love Waves in an Elastic Layer Overlying a Rigid Basement, Proc. of JSCE Structural Eng./Earthquake Eng. Vol.2, No. 1, pp. 227 235, April 1985.

2) Clough, R.W. and Penzien, J. : Dynamics of Structures, McGraw-Hill Kogakusha, Ltd., pp. 195 198, 1975.

3) Knopoff, L. : Q, Review of Geophysics, Vol.2, No.4, pp.625 660, Nov. 1964.

4) Alsop, L. E. : Transmission and Reflection of Love Waves at a Vertical Discontinuity, Journal of Geophysical Research, Vol. 71, No. 16, pp. 3969 3984, 1966.

5) Harkrider, D. G. : Surface Waves in Multilayered Elastic Media I. Rayleigh and Love Waves from Buried Sources in a Multilayered Elastic Half-space, Bull. Seis. Soc. Am., Vol. 54, No. 2, pp. $627 \sim 679,1964$.

6) Harkrider, D. G. and Anderson, D. L. : Surface Wave Energy from Point Sources in Plane Layered Earth Models, Journal of Geophysical Research, Vol.71, No. 12, pp. 2967 2980, 1966.

7) Harkrider, D. G. : Surface Waves in Multilayered Elastic Media. Part II. Higher Mode Spectra and Spectral Ratios from Point Sources in Plane Layered Earth Models, Bull. Seis. Soc. Am., Vol.60, No. 6, pp. 1937 1987, 1970.

8) Tazime, K. : Minimun Group Velocity, Maximum Amplitude and Quater Wave-length Law. Love-waves in Doubly Stratified 
